\title{
Inhibition of AKT survival pathway by a small molecule inhibitor in human endometrial cancer cells
}

\author{
X Jin', DR Gossett' ${ }^{2}$ S Wang ${ }^{3}$, D Yang ${ }^{3}$, Y Cao ${ }^{3}$, J Chen ${ }^{3}$, R Guo ${ }^{3}$, RK Reynolds ${ }^{2}$ and J Lin ${ }^{*, 4}$ \\ 'Department of Pathology, University of Michigan Comprehensive Cancer Center, Ann Arbor, MI, USA; ${ }^{2}$ Department of Obstetrics and Gynecology, \\ University of Michigan Comprehensive Cancer Center, Ann Arbor, MI, USA; ${ }^{3}$ Division of Hematology and Oncology, Department of Internal Medicine, \\ University of Michigan Comprehensive Cancer Center, Ann Arbor, MI, USA; ${ }^{4}$ Center for Childhood Cancer, Columbus Children's Research Institute, The \\ Ohio State University, Columbus, OH 43205, USA
}

The PTEN (phosphatase and tensin homolog deleted on chromosome 10) tumour suppressor is mutated in 40-50\% of human endometrial cancers. PTEN exerts its effects in part via inhibition of the antiapoptotic protein AKT. We demonstrate that two endometrial cancer cell lines that harbour PTEN mutations, Ishikawa and RL95-2, have high levels of phosphorylated AKT and high AKT kinase activity. Two additional endometrial cancer cell lines that express wild-type PTEN, HeclA and KLE, have little phosphorylated AKT and minimal demonstrable AKT kinase activity. We tested a potential inhibitor of the AKT pathway, API-59CIOMe, in these four cell lines. We found that API-59CJ-OMe inhibits AKT kinase activity and induces apoptosis in the Ishikawa and RL95-2 cell lines with high AKT activity, but has little effect on HecIA and KLE cells without AKT activity. API-59CJ-OMe may therefore have therapeutic potential for those endometrial cancers that harbour PTEN mutations and AKT activation.

British Journal of Cancer (2004) 91, I808-18|2. doi:10.1038/sj.bjc.66022I4 www.bjcancer.com

Published online 26 October 2004

(c) 2004 Cancer Research UK

Keywords: AKT; PTEN; endometrial cancer; apoptosis; experimental therapeutics

Endometrial cancer is the most common gynaecologic malignancy in developed countries, with approximately 40000 new diagnoses each year in the US alone. Mutations of the tumour suppressor PTEN are found in $40-50 \%$ of human endometrial cancers (Tashiro et al, 1997; Ali, 2000). PTEN is also frequently mutated in brain, breast, and prostate cancers (Cairns et al, 1997; Li and Sun, 1998; Davies et al, 1999). PTEN is thought to function as a tumour suppressor due to its ability to block $G_{1}$ cell cycle progression, induce apoptosis, and negatively regulate the PI3-K/ AKT cell survival pathway (Stambolic et al, 1998). Accordingly, in cancer cells with PTEN mutation or deletion, AKT activity is dramatically elevated (Kanamori et al, 2001). AKT is a serine/ threonine kinase that is activated in response to growth factors or cytokines by a mechanism involving phosphoinositide 3-kinase (PI3-K) and phosphoinositide-dependent kinase-1 (PDK-1) (Franke et al, 1997; Kulik et al, 1997). AKT has three isoforms: $\mathrm{AKT} 1(\mathrm{PKB} \alpha), \mathrm{AKT} 2(\mathrm{PKB} \beta)$, and AKT3 (PKB $\gamma)($ Datta et al, 1999). These isoforms have greater than $85 \%$ sequence identity and have the same structural organisation. Amplification of the AKT2 oncogene and high AKT kinase activity have been detected in breast, pancreatic, ovarian, brain, prostate, and gastric cancers (Cheng et al, 1992; Bellacosa et al, 1995).

\footnotetext{
*Correspondence: J Lin, Center for Childhood Cancer, Columbus Children's Research Institute, The Ohio State University, Columbus, $\mathrm{OH}$ 43205, USA; E-mail: linj@pediatrics.ohio-state.edu

Received 24 May 2004; revised 24 August 2004; accepted 13 September 2004; published online 26 October 2004
}

AKT provides a survival signal that protects cells from apoptosis induced by various stresses (Franke et al, 1997; Kulik et al, 1997). Some examples of the known mechanisms by which AKT prevents apoptosis are the phosphorylation of Bad, glycogen synthase kinase-3 (GSK-3), forkhead transcription factor (FKHR), and caspase-9 (Del Peso et al, 1997; Cardone et al, 1998; Pap and Cooper, 1998; Brunet et al, 1999). Phosphorylation of these proteins results in inactivation of their apoptotic functions. AKT may suppress apoptosis by stimulating the transactivation potential of the RelA/p65 subunit of NF-kappaB (Romashkova and Makarov, 1999; Madrid et al, 2000). AKT may also enhance the ubiquitination-promoting function of $\mathrm{Mdm} 2$, resulting in ubiquitin-mediated degradation of the tumour suppressor p53 (Ogawara et al, 2002).

Development of potent AKT inhibitors is a promising therapeutic strategy for endometrial carcinoma. We have employed a bioinformatics approach to identify potential inhibitors of the AKT pathway (Wang and Yang, manuscript in preparation). We first performed a Western blot analysis to probe the level of phosphorylated AKT (p-AKT) in the National Cancer Institute (NCI) 60 human cancer cell lines. Correlation analysis was performed of the in vitro anticancer activity of 35000 compounds in the NCI's anticancer database, and the p-AKT levels in the NCI 60 human cancer cell lines to identify compounds whose in vitro anticancer activities significantly correlated with the p-AKT level in the 60 cancer cell lines. Compounds whose in vitro anticancer activities significantly correlated with the p-AKT level in the 60 cancer cell lines were considered as candidate inhibitors for the AKT pathway. API-59CJ-OMe (9-methoxy-2-methylellipticinium acetate) was identified as a potential inhibitor. Our further 
evaluations in human prostate and breast cancer cell lines showed that API-59CJ-OMe potently inhibits cell growth and induces apoptosis in cell lines with high levels of p-AKT, but has minimal activity in cell lines with low levels of p-AKT (Wang and Yang, manuscript in preparation), suggesting that API-59CJ-OMe may target the AKT pathway.

In the present study, we tested API-59CJ-OMe in PTEN-defective endometrial cancer cells. We found that API-59CJ-OMe selectively inhibits AKT kinase activity and induces apoptosis in endometrial cancer cell lines expressing high levels of AKT activity. API-59CJ$\mathrm{OMe}$ has little effect in endometrial cancer cells lacking AKT activity. This is the first report of a potential AKT inhibitor in endometrial cancer.

\section{MATERIALS AND METHODS}

\section{Cell lines}

Hec1A, RL95-2, and KLE human endometrial cancer cell lines were purchased from American Type Culture Collection (Manassas, VA, USA). Ishikawa human endometrial cancer cell line has been previously described and was obtained from Dr Masato Nishida (Holinka et al, 1986). Cells were maintained in 90\% Dulbecco's modified Eagle's medium supplemented with $10 \%$ fetal bovine serum and antibiotics (5000 $\mathrm{U} \mathrm{ml}^{-1}$ penicillin G, $5000 \mu \mathrm{g} \mathrm{ml}^{-1}$ streptomycin) (Gibco/BRL Life Technologies, Inc., Rockville, MD, USA) at $37^{\circ} \mathrm{C}$ in $5 \% \mathrm{CO}_{2}$.

\section{Western blot analysis}

To analyse levels of phosphorylation of AKT, PDK1, and ERK1/2 proteins, cells were plated at $1.2 \times 10^{6}$ cells. $100 \mathrm{~mm}^{-1}$ dish 1 day prior to treatment. Cells were then treated with API-59CJ-OMe at the indicated dose for 48 or $72 \mathrm{~h}$ before harvesting. In total, $100 \mu \mathrm{g}$ of total protein from cell lysates was separated on 10\% SDSpolyacrylamide gels and blotted with a $1: 1000$ dilution of antibodies against phospho-AKT (Ser 473 or Thr 308), phosphoPDK1 (Ser 241), phospho-ERK1/2 (Thr202/Tyr204), phospho-JNK (Thr 183/Tyr 185), phospho-SGK (Ser 78), phospho-PKC $\zeta / \lambda$ (Thr 410/403), phospho-PKC $\alpha / \beta$ (Thr 638/641), total AKT, or total PTEN (Cell Signaling Tech., Beverly, MA, USA). The same membranes were analysed with a $1: 2500$ dilution of anti-GAPDH (glyceraldehyde-3-phosphate dehydrogenase) monoclonal antibody (Chemicon International, Inc., Temecula, CA, USA) as a protein loading control. All blots were incubated with 1:10000 dilution of secondary alkaline phosphatase-conjugated anti-mouse or anti-rabbit antibody (Amersham Pharmacia Biotech., Piscataway, NJ, USA). Blots were scanned with ImageQuant Software using an ECF Western blotting detection system (Amersham Pharmacia Biotech., Piscataway, NJ, USA) on a Molecular Dynamics STORM PhosphorImager (Sunnyvale, CA, USA). Each Western blot was performed a minimum of three times.

\section{AKT and ERK kinase assays}

RL95-2 and Ishikawa cells were seeded at $1.2 \times 10^{6}$ cells in 100 $\mathrm{mm}$ dishes for $24 \mathrm{~h}$ before treatment. Cells were then exposed to API-59CJ-OMe at 12 or $24 \mu \mathrm{M}$ for $48-72 \mathrm{~h}$. Protein $(500 \mu \mathrm{g})$ from cell lysates was immunoprecipitated with either anti-AKT monoclonal antibody or anti-ERK monoclonal antibody. AKT kinase assays and extracellular signal-regulated kinases (ERK) kinase assays were performed using AKT and ERK kinase assay kits (Cell Signaling Tech., Beverly, MA, USA) with glycogen synthase kinase3 (GSK-3) as a substrate for AKT and Elk-1 as a substrate for ERK. Phospho-specific antibodies to GSK-3 $\alpha / \beta$ (Ser 21/9) and Elk-1 (Ser 383) were used for phosphorylated protein detection. At least three independent repetitions were performed for each assay type. An additional assay was performed to verify equal immunoprecipita-

tion of AKT from treated and untreated cells; AKT was immunoprecipitated from cell lysates using the same anti-AKT monoclonal antibody as described above. Cell lysates were incubated and washed as per the kinase assay protocol, but then separated via Western blot prior to the performance of the kinase assay.

\section{Apoptotic assay}

API-59CJ-OMe was synthesised in Dr Shaomeng Wang's laboratory at the University of Michigan. To quantitate the induction of apoptosis by API-59CJ-OMe, cells from all four cell lines were plated at $3 \times 10^{5}$ cells per 6 - $\mathrm{cm}$ dish 1 day prior to exposure to API-59CJ-OMe at $1.5,6,12$, or $24 \mu \mathrm{M}$. After $72 \mathrm{~h}$ of treatment, cells were harvested and fixed with $70 \%$ ethanol. Cells were then stained with propidium iodide and analysed for reduction of DNA content (sub-G1 profile) on a FACScan flow cytometer (Becton Dickinson, San Jose, CA, USA). The results given are average values and standard deviations from at least three separate experiments.

\section{RESULTS}

\section{Expression of PTEN protein and phosphorylated AKT in endometrial cancer cell lines}

We first examined the expression of PTEN and phosphorylation of AKT in the four human endometrial cancer cell lines, RL95-2, Ishikawa, Hec1 A, and KLE. Two of these cell lines, HeclA and KLE, express a considerable amount of PTEN protein, whereas the other two, Ishikawa and RL95-2, express little or no PTEN protein

AKT kinase assay
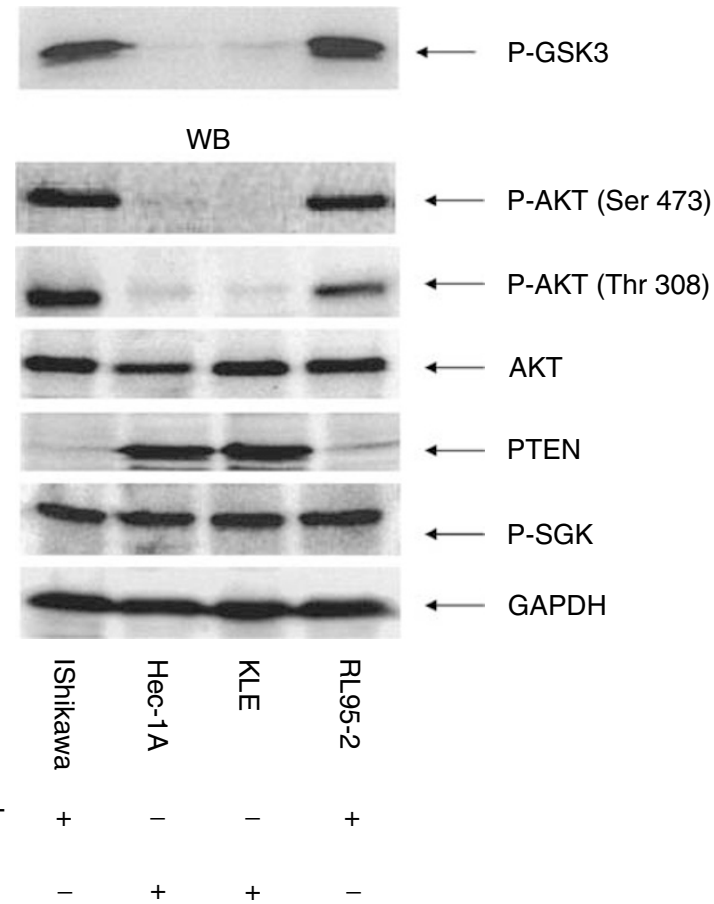
(Figure 1). This is consistent with previous reports that Ishikawa and RL95-2 cell lines contain PTEN mutations, while Hec1A and KLE cells express wild-type PTEN (Yaginuma et al, 2000). We also found that Ishikawa and RL95-2 cell lines express high levels of phosphorylated AKT and have high AKT kinase activity, whereas Hec1A and KLE cells express little phosphorylated AKT and have undetectable AKT kinase activity (Figure 1). However, phosphorylation of serum- and glucocorticoid-inducible kinase (SGK), an AKT-related serine/threonine kinase, is independent of PTEN status in these cancer cell lines (Figure 1). Therefore, in these four cell lines, there is perfect correlation between loss of PTEN expression and overactivation of AKT kinase.

\section{Inhibition of AKT kinase activity in human endometrial cancer cell lines}

Through a bioinformatics approach, we have identified a nonpeptide small molecule inhibitor, API-59CJ-OMe, as a potential inhibitor of the AKT pathway (Wang and Yang, manuscript in preparation). The chemical structure of API-59CJ-OMe is shown in Figure 2. We tested whether API-59CJ-OMe can inhibit AKT kinase activity in RL95-2 and Ishikawa cells, the two cell lines with high

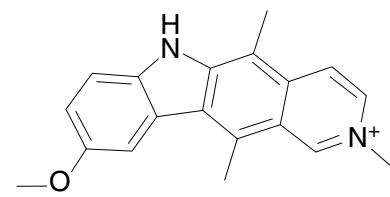

Figure 2 Chemical structure of API-59CJ-OMe, 9-methoxy-2-methylellipticinium acetate. levels of AKT phosphorylation and kinase activity. Addition of API-59CJ-OMe significantly inhibited AKT kinase activity when GSK-3 is used as a substrate in RL95-2 and Ishikawa cells (Figure 3). The amount of total AKT immunoprecipitated from cell lysates was not affected by treatment with API-59CJ-OMe. API59CJ-OMe had no effect on ERK kinase activity. To further demonstrate the selectivity of API-59CJ-OMe, we probed the same cell lysates with antibodies against phosphorylated MAP kinases (ERK1/2 and JNK1/2), phosphorylated AKT, phosphorylated SGK, phosphorylated PKC isoforms, or phosphorylated PDK-1. As shown in Figure 3, API-59CJ-OMe did not inhibit phosphorylation of these proteins. Equal protein loading was demonstrated by blotting the same membranes with GAPDH antibody. API-59CJOMe did not affect kinases either upstream of AKT (PDK-1) or in a distinct signal transduction pathway (ERK1/2 and JNK1/2) in endometrial cancer cells. Of note, API-59CJ-OMe did not inhibit phosphorylation of AKT itself at either Serine 473 or Threonine 308. This suggests that API-59CJ-OMe may act at the AKT kinase level and is less likely to act upstream at kinases responsible for either Serine 473 or Threonine 308 phosphorylation.

\section{Induction of apoptosis in human endometrial cancer cell lines with elevated AKT activity}

We examined whether API-59CJ-OMe could induce apoptosis in RL95-2 and Ishikawa endometrial cancer cell lines. We predicted that inhibition of the AKT survival pathway by API-59CJ-OMe would lead to apoptosis of these cancer cells. As expected, exposure of API-59CJ-OMe at 12 and $24 \mu \mathrm{m}$ significantly induced apoptosis in both RL95-2 and Ishikawa endometrial cancer cell lines, which express high levels of phosphorylated AKT and AKT kinase activity. API-59CJ-OMe had only minimal effects on Hec1 A
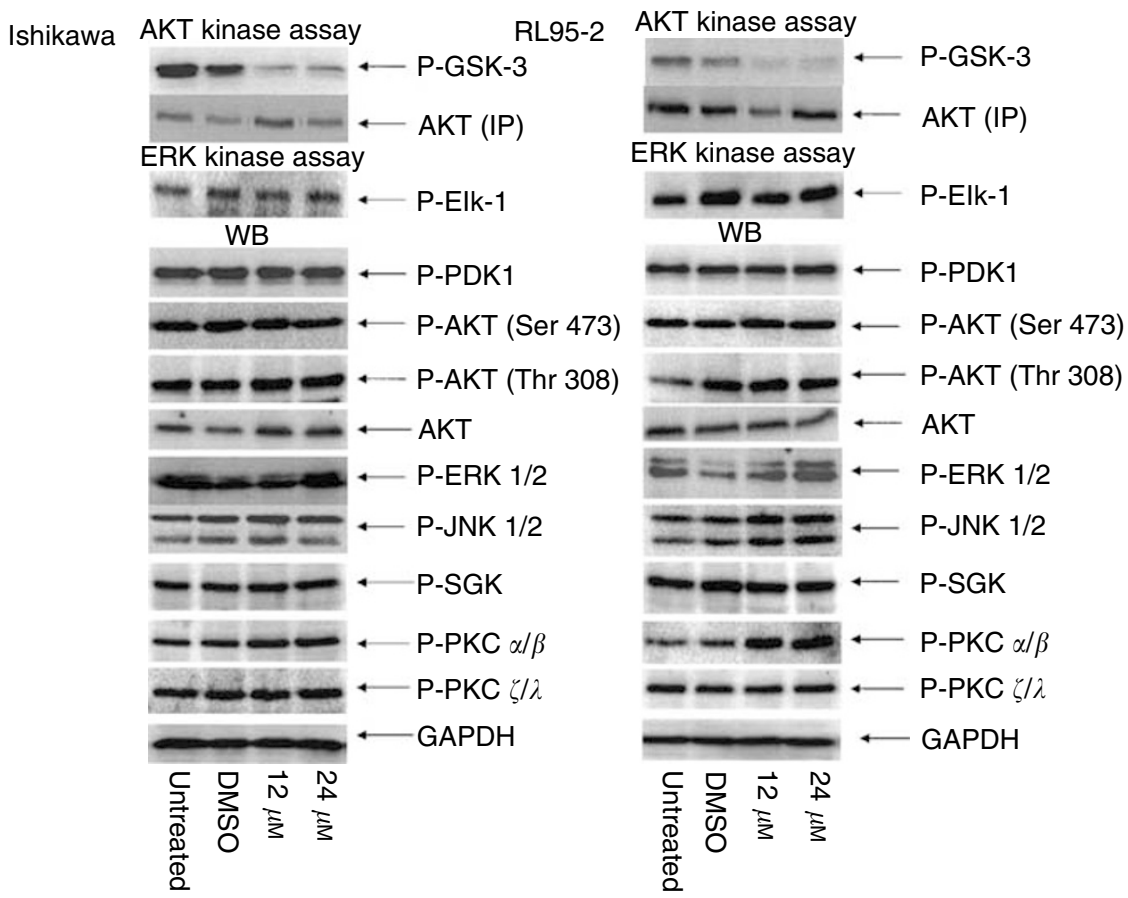

Figure 3 Effect of API-59CJ-OMe on AKT kinase activity and phosphorylation of other kinases. (A) After treatment with API-59CJ-OMe, cells were harvested and cell lysates separated by 10\% SDS-PAGE. Gels were analysed with phospho-specific antibodies to PDKI (Ser 24I), AKT (Ser 473 or Thr 308), ERKI/2 (Thr 202/Tyr 204), JNK (Thr I83/ Tyr 185), SGK (Ser 78), PKC $\zeta / \lambda$ (Thr 4I0/403), and PKC $\alpha / \beta$ (Thr 638/64I). The same lysates were immunoprecipitated with anti-AKT or anti-ERK antibody, and kinase assays performed with GSK-3 as a substrate for AKT and Elk-I as a substrate for ERK AKT (IP) represents the immunoprecipitation control: lysates were immunoprecipitated with mouse monoclonal anti-AKT antibody, separated by SDSPAGE, and stained with rabbit polyclonal anti-AKT antibody. Phospho-specific antibodies to GSK-3 $\alpha / \beta$ (Ser 21/9) and Elk-I (Ser 383) were used for phosphorylated protein detection. 
A
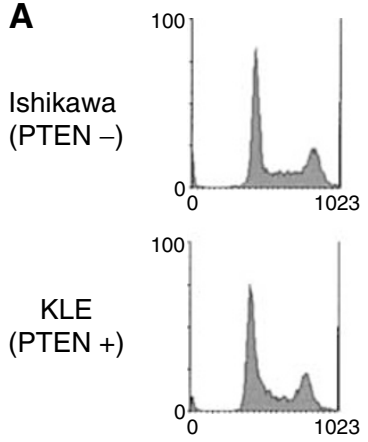

Untreated $(0 \mu \mathrm{M})$
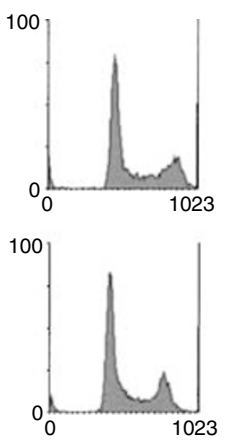

DMSO
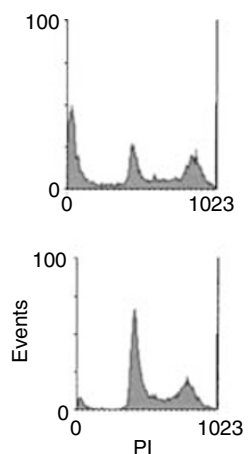

API-59

$12 \mu \mathrm{M}$
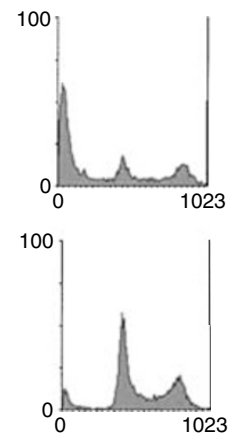

B

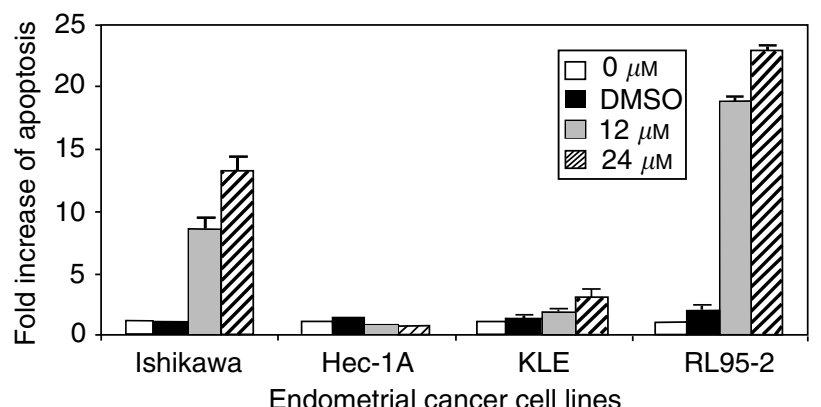

Figure 4 API-59CJ-OMe induces apoptosis of endometrial cancer cells expressing activated AKT. (A) Two examples of flow cytometry histograms in Ishikawa and KLE cells; API-59CJ-OMe induced marked apoptosis in Ishikawa and RL95-2 cells, but not in Hecl A or KLE cells. (B) Fold increase of apoptosis was calculated as percentage apoptosis in treated cells over percentage apoptosis in untreated cells. Results shown in (B) are the average and standard deviation of Log PI from three independent experiments.

and KLE, cell lines that express wild-type PTEN and lack AKT kinase activity (Figure 4). Lower doses (1.5 and $6 \mu \mathrm{M}$ ), when screened using MTT cell viability assays, had minimal effects on cell number, and were significantly less effective at inducing apoptosis in flow cytometry assays (data not shown).

\section{DISCUSSION}

PTEN mutation is a frequent event in human endometrial cancer. Good correlation exists between loss of PTEN expression and high levels of phosphorylated AKT in endometrial cancer cells (Kanamori et al, 2001). High AKT kinase activity in cancer cells may provide survival or proliferation signals and promote oncogenesis (Franke et al, 1997; Kulik et al, 1997). Thus, inhibition of AKT represents an attractive therapy for the treatment of endometrial cancer. Inhibitors that target upstream regulators of AKT such as PI3-K and PDK-1, or potentially target the pleckstrin homology (PH) domain of AKT, have been reported ( $\mathrm{Hu}$ et al, 2000; Ballif et al, 2001; Kozikowski et al, 2003; Meuillet et al, 2003). Introduction of wild-type PTEN into endometrial cancer cells harbouring PTEN mutation can inhibit cell growth (Lilja et al, 2001). In this report, we describe a potential small molecule inhibitor of the AKT pathway. Small molecule drugs have several advantages, including good delivery properties, good in vivo stability, low probability of immune response, and low cost.

API-59CJ-OMe (or MMEA) belongs to the class of compounds referred to as ellipticines. Ellipticines with a wide variety of substitutions at the 2 and 9 positions have been evaluated as potential antitumour agents, and there is evidence for several different mechanisms of action for this group of drugs. The ellipticines can bind directly to DNA, and the 2, 9 substituted ellipticines may act as DNA 'threaders', intercalating into the DNA strands (Harding and Grummitt, 2003). These compounds also stabilise topoisomerase II-DNA complexes, and promote DNA strand breakage. Some studies have also suggested that the ellipticines may act to restore wild-type p53 function in cells with mutant p53, although the results in our lab do not confirm this. Finally, some of the ellipticines (9-hydroxyellipticines) may promote generation of superoxide radicals, leading to DNA strand breakage. Exactly how these described mechanisms relate to the AKT inhibition we see in our system is still unclear, and we are working further to clarify this. Several compounds have been used in Phase I and II human trials for a variety of malignancies. The compound appears to be metabolised by cytochrome P450, and reported toxicities have varied widely across trials, which have mostly involved 9-hydroxy compounds. There is in vitro data that suggests that API-59CJ-OMe/MMEA may display some neurotoxicity (Sriram et al, 1997). Additional testing to determine the toxicity profiles of API-59-CJOMe will be required before this compound can be considered as a possible human therapeutic. The lack of major effects on the two PTEN-intact cell lines suggests that normal cells may have some innate resistance to the compound. However, this will have to be verified in animal models, as cell culture has only limited capacity to predict in vivo toxicity.

Our data demonstrate that API-59CJ-OMe can inhibit AKT kinase activity in cell culture. API-59CJ-OMe does not inhibit ERK kinase, nor did it affect phosphorylation of ERK1/2, JNK1/2, PKC isoforms, SGK, PDK1, or AKT itself. This suggests that API-59CJOMe may inhibit the AKT pathway at the AKT level, but not at upstream kinases that phosphorylate AKT at Serine 473 or Threonine 308, in endometrial cancer cells. API-59CJ-OMe induces apoptosis in endometrial cancer cell lines expressing high levels of AKT activity, but has little effect in endometrial cancer cells lacking AKT activity. Further, both Ishikawa and RL95-2 cells 
harbour endogenous mutant p53 and API-59CJ-OMe did not induce p53 targets, mdm2 (data not shown). This suggests that API-59CJ-OMe does not induce apoptosis through the p53dependent pathway. AKT is frequently activated in endometrial carcinoma due to PTEN mutation, API-59CJ-OMe has potential clinical applications in endometrial cancer. We plan to further explore inhibition of AKT pathway using this small molecule inhibitor in a nude mouse xenograft model.

In addition to endometrial cancer, PTEN is frequently mutated in brain and prostate cancer (Cairns et al, 1997; Li and Sun, 1998). AKT activity is also elevated in many ovarian and breast cancers. In these tumours, AKT activation is mainly due to amplification of the $A K T$ oncogene or activation by upstream regulators (Cheng et al, 1992; Bellacosa et al, 1995; Tashiro et al, 1997). It will be of interest to determine whether API-59CJ-OMe can inhibit other cancer types with high AKT kinase activity. Our lab has already shown that API-59CJ-OMe inhibits AKT kinase activity and induces apoptosis in ovarian cancer cells with high AKT activity
(Tang and Lin, manuscript submitted). These studies represent the first steps towards developing a small molecule therapy that targets the AKT oncogenic pathway. This approach may prove useful not only against endometrial cancer but against prostate cancer, brain cancer, and other tumours where disruption of PTEN and AKT is common.

\section{ACKNOWLEDGEMENTS}

We thank Dr Masato Nishida at the Kasumigaura National Hospital in Japan for providing the Ishikawa endometrial cancer cell line. This work was supported in part by the Samuel Waxman Cancer Research Foundation. Dr Gossett is a SGI/NICHD Fellow of the Reproductive Scientist Development Programme, funded through NIH Grant \#2K12HD00849 and a Wyeth-Ayherst Pharmaceuticals grant.

\section{REFERENCES}

Ali I (2000) Gatekeeper for endometrium: the PTEN tumor suppressor gene. J Natl Cancer Inst 92: $861-863$

Ballif B, Shimamura A, Pae E, Blenis J (2001) Disruption of 3phosphoinositide-dependent kinase 1 (PDK1) signaling by the antitumorigenic and anti-proliferative agent $n$-alpha-tosyl-l-phenylalanyl chloromethyl ketone. J Biol Chem 276: 12466-12475

Bellacosa A, de Feo D, Godwin A, Bell D, Cheng J, Altomare D, Wan M, Dubeau L, Scambia G, Masciullo V, Ferrandina G, Panici P, Mancuso S, Neri G, Testa J (1995) Molecular alterations of the AKT2 oncogene in ovarian and breast carcinomas. Int J Cancer 64: 280-285

Brunet A, Bonni A, Zigmond M, Lin M, Juo P, Hu L, Anderson M, Arden K, Blenis J, Greenberg M (1999) Akt promotes cell survival by phosphorylating and inhibiting a Forkhead transcription factor. Cell 96: 857-868

Cairns P, Okami K, Halachmi S, Halachmi N, Esteller M, Herman J, Jen J, Isaacs W, Bova G, Sidransky D (1997) Frequent inactivation of PTEN/ MMAC1 in primary prostate cancer. Cancer Res 57: 4997-5000

Cardone M, Roy N, Stennicke H, Salvesen G, Franke T, Stanbridge E, Frisch S, Reed J (1998) Regulation of cell death protease caspase-9 by phosphorylation. Science 282: $1318-1321$

Cheng J, Godwin A, Bellacosa A, Taguchi T, Franke T, Hamilton T, Tsichlis $\mathrm{P}$, Testa J (1992) AKT2, a putative oncogene encoding a member of a subfamily of protein-serine/threonine kinases, is amplified in human ovarian carcinomas. Proc Natl Acad Sci USA 89: 9267 - 9271

Datta SR, Brunet A, Greenberg ME (1999) Cellular survival: a play in three AKTs. Genes Dev 13: 2905-2927

Davies M, Koul D, Dhesi H, Berman R, McDonnell T, McConkey D, Yung W, Steck P (1999) Regulation of Akt/PKB activity, cellular growth, and apoptosis in prostate carcinoma cells by MMAC/PTEN. Cancer Res 59: $2551-2556$

Del Peso L, Gonzalez-Garcia M, Page C, Herrera R, Nunez G (1997) Interleukin-3-induced phosphorylation of $\mathrm{BAD}$ through the protein kinase Akt. Science 278: 687-689

Franke T, Kaplan D, Cantley L (1997) PI3K: downstream AKTion blocks apoptosis. Cell 88: $435-437$

Harding M, Grummitt A (2003) 9-Hydroxyellipticine and derivatives as chemotherapy agents. Mini Rev Med Chem 3: 67-76

Holinka C, Hata H, Kuramoto H, Gurpide E (1986) Effects of steroid hormones and antisteroids on alkaline phosphatase activity in human endometrial cancer cells (Ishikawa line). Cancer Res 46: 2771-2774

$\mathrm{Hu}$ L, Zaloudek C, Mills G, Gray J, Jaffe $\mathrm{R}$ (2000) In vivo and in vitro ovarian carcinoma growth inhibition by a phosphatidylinositol 3-kinase inhibitor (LY294002). Clin Cancer Res 6: 880-886

Kanamori Y, Kigawa J, Itamochi H, Shimada M, Takahashi M, Kamazawa S, Sato S, Akeshima R, Terakawa N (2001) Correlation between loss of

PTEN expression and Akt phosphorylation in endometrial carcinoma. Clin Cancer Res 7: $892-895$

Kozikowski A, Sun H, Brognard J, Dennis P (2003) Novel PI analogues selectively block activation of the pro-survival serine/threonine kinase Akt. J Am Chem Soc 125: $1144-1145$

Kulik G, Klippel A, Weber MJ (1997) Antiapoptotic signalling by the insulin-like growth factor I receptor, phosphatidylinositol 3-kinase, and Akt. Mol Cell Biol 17: 1595-1606

Li D, Sun H (1998) PTEN/MMAC1/TEP1 suppresses the tumorigenicity and induces G1 cell cycle arrest in human glioblastoma cells. Proc Natl Acad Sci USA 95: 15406-15411

Lilja J, Wu D, Reynolds R, Lin J (2001) Growth suppression activity of the PTEN tumor suppressor gene in human endometrial cancer cells. Anticancer Res 21: $1969-1974$

Madrid L, Wang C, Guttridge D, Schottelius A, Baldwin AJ, Mayo M (2000) Akt suppresses apoptosis by stimulating the transactivation potential of the RelA/p65 subunit of NF-kappaB. Mol Cell Biol 20: 1626-1638

Meuillet E, Mahadevan D, Vankayalapati H, Berggren M, Williams R, Coon A, Kozikowski A, Powis G (2003) Specific inhibition of the Akt1 pleckstrin homology domain by D-3-deoxy-phosphatidyl-myo-inositol analogues. Mol Cancer Ther 2: 389-399

Ogawara Y, Kishishita S, Obata T, Isazawa Y, Suzuki T, Tanaka K, Masuyama N, Gotoh Y (2002) Akt enhances Mdm2-mediated ubiquitination and degradation of p53. J Biol Chem 277: 21843-21850

Pap M, Cooper G (1998) Role of glycogen synthase kinase-3 in the phosphatidylinositol 3-kinase/Akt cell survival pathway. J Biol Chem 273: 19929 - 19932

Romashkova J, Makarov S (1999) NF-kappaB is a target of AKT in antiapoptotic PDGF signalling. Nature 401: 86-90

Sriram K, MR B, Vistica D, Ravindranath R (1997) In vitro neurotoxicity of the antitumor agent 9 -methoxy- $N_{2}$-methylellipticinium acetate (MMEA): role of brain cytochrome P-450. Neurotoxicity 18: $97-104$

Stambolic V, Suzuki A, de la Pompa J, Brothers G, Mirtsos C, Sasaki T, Ruland J, Penninger J, Siderovski D, Mak T (1998) Negative regulation of PKB/Akt-dependent cell survival by the tumor suppressor PTEN. Cell 95: 29-39

Tashiro H, Blazes M, Wu R, Cho K, Bose S, Wang S, Li J, Parsons R, Ellenson L (1997) Mutations in PTEN are frequent in endometrial carcinoma but rare in other common gynecological malignancies. Cancer Res 57: 3935 - 3940

Yaginuma Y, Yamashita T, Ishiya T, Morizaki A, Katoh Y, Takahashi T, Hayashi H, Ishikawa M (2000) Abnormal structure and expression of PTEN/MMAC1 gene in human uterine cancers. Mol Carcinog 27: $110-116$ 